\title{
The CC Genotype of Insulin-Induced Gene 2 rs7566605 Is a Protective Factor of Hypercholesteremia Susceptible to Mild Cognitive Impairment, Especially to the Executive Function of Patients with Type 2 Diabetes Mellitus
}

\author{
Haoqiang Zhang $\mathbb{D}^{1,2}$ Rong Huang, ${ }^{1,2}$ Sai Tian, ${ }^{1,2}$ Ke An, ${ }^{1,2}$ Wenwen Zhu, ${ }^{1,2}$ Jijing Shi, ${ }^{1,2}$ \\ Wuyou Cao, ${ }^{1,2}$ and Shaohua Wang ${ }^{1,2}$ \\ ${ }^{1}$ Department of Endocrinology, Affiliated Zhongda Hospital of Southeast University, No. 87 Dingjiaqiao Road, Nanjing, China \\ 210009 \\ ${ }^{2}$ School of Medicine, Southeast University, Nanjing, China 210009
}

Correspondence should be addressed to Shaohua Wang; gyjwsh@126.com

Received 27 December 2019; Revised 4 April 2020; Accepted 28 May 2020; Published 10 June 2020

Academic Editor: Margaret A. Niznikiewicz

Copyright (c) 2020 Haoqiang Zhang et al. This is an open access article distributed under the Creative Commons Attribution License, which permits unrestricted use, distribution, and reproduction in any medium, provided the original work is properly cited.

\begin{abstract}
Backgrounds and Aims. Insulin-induced gene 2 (INSIG-2) is closely related to hypercholesteremia, which is a well-recognized risk factor of mild cognitive impairment (MCI) in type 2 diabetes mellitus (T2DM). We aim to investigate the association between promoter of the INSIG-2 rs7566605 single-nucleotide polymorphism (SNP) and T2DM with MCI. Methods. 233 T2DM patients with MCI or without MCI were recruited. Baseline data and genotype frequency were compared between MCI and non-MCI groups. Demographic parameters and neuropsychological tests results were analyzed among patients with different genotypes. Further correlation and regression analysis were conducted to find the association between cognition and cholesterol. Results. Despite no significant statistical difference was detected, we observed higher levels of total cholesterol (TC) and low-density lipoprotein cholesterol (LDL) in patients with MCI than those without MCI. In addition, we observed higher TC and LDL levels in patients with GG or GC genotypes than those with CC genotype $(P<0.001, P=0.004$, or $P<0.001, P=0.002)$. Interestingly, increased MoCA and decreased TMTB scores were found in patients with CC genotype, compared to those with GG or CG genotype $(P=0.009, P=0.024$, or $P=0.005, P=0.109)$. Moreover, partial correlation $(P=0.030$ and $P=0.004$, respectively) and multiple linear regression $(P=0.030$ and $P=0.005$, respectively) showed that TC and LDL levels are associated with the TMTB score, indicating the executive function. Conclusions. CC genotype of INSIG-2 rs7566605 may be a protective factor of hypercholesteremia susceptible to MCI, especially to the executive function of T2DM. This trial is registered with ChiCTROCC15006060.
\end{abstract}

\section{Introduction}

The prevalence of type 2 diabetes mellitus among adults has increased to $10.4 \%$ and affected 300 million worldwide [1]. Diabetes increased about 50\% risk of Alzheimer's disease $(\mathrm{AD})$ [2] with a prodromal stage called MCI, a transitional stage between normal cognition and $\mathrm{AD}$ dementia [3]. Previous researches suggested that uncontrolled hyperglycemia is a risk factor of T2DM with MCI $[4,5]$. Epide- miological evidence showed that cholesterol is also a risk factor for $\mathrm{AD}$ [6]. In addition, cholesterol homeostasis failure is a cause of synaptic degeneration [7]. Our recent study demonstrated that poorly controlled cholesterol is associated with cognitive impairment in T2DM human [8]. Moreover, increased LDL cholesterol caused cognitive decline in $L D L^{-/-}$mice [9]. So, we guess that the reason causing hypercholesterolemia may result in cognitive impairment. 
Insulin induced genes (INSIGs), including INSIG-1 [10] and INSIG-2 [11], and sensors and mediators that regulate cholesterol homeostasis through sterol regulatory elementbinding proteins (SREBPs) and SREBP cleavage-activating protein (SCAP), are discovered and regarded as crucial roles in cholesterol metabolism. INSIGs could negatively regulate 3-hydroxy-3-methylglutaryl coenzyme A reductase (HMGCoA reductase), an important key enzyme of cholesterol synthesis [12]. So, we hypothesize that INSIG-2 plays an important role in cognitive impairment via regulating cholesterol homeostasis.

There is an interesting SNP; rs7566605, which is located $10 \mathrm{~kb}$ upstream of INSIG-2, was found to be associated with obesity, assessed by BMI [13]. However, Wang et al. [14] had reported that $r s 7566605$ may be not associated with severe obesity in Chinese children. Although there was no association found between genotype at rs7566605 and obesity-related phenotypes in this British Caucasian population [15], the promoter of INSIG-2 rs7566605 SNP is associated with the prevalence of hypercholesterolemia [16]. Additionally, the CC genotype is an independent protective genetic factor for the progressing of hypercholesterolemia induced by high-fat diet, especially in female subjects. Zavattari et al. [17] conducted a study, which suggested that the INSIG-2 rs7566605 SNP may play a role in metabolic complications related to obesity in obese children and adolescents. An intervention study showed that $r 57566605$ SNP of INSIG-2 had effects on weight change [18]. However, the relationship among rs7566605 SNP of INSIG-2, hypercholesterolemia, and mild cognitive impairment remains unclear in patients with type 2 diabetes mellitus. In the present study, we aim to investigate the potential protective role of $\mathrm{CC}$ genotype of INSIG-2 in cognitive decline.

\section{Materials and Methods}

2.1. Ethics. This present study was conducted in the Endocrinology Department, Affiliated Zhongda Hospital of Southeast University. All individuals were hospitalized patients and provided a written informed consent prior to the participation of this study. The present study was approved by the Research Ethics Committee, Affiliated Zhongda Hospital of Southeast University.

2.2. Subjects and Groups. This cross-sectional study recruited 233 (87 T2DM with MCI and 146 T2DM without MCI) hospitalized patients who satisfied the criteria of type 2 diabetes mellitus [19]. All individuals were right-handed Han Chinese having diabetes for more than 3 years. The diagnostic criteria for MCI were proposed by the MCI Working Group of the European Consortium for Alzheimer's Disease [20]. The individuals were included in or excluded from this study according to the criteria of our previous studies [21].

2.3. Clinical Data. The following demographic characteristics were gathered: age, gender, and education. Duration of diabetes mellitus (DM) and high blood pressure (HBP) were recorded. Medication histories of metformin and insulin were collected. Body mass index (BMI) was calculated.
Fasting blood-glucose (FBG), glycosylated hemoglobin (HbAlc), triglyceride (TG), TC, LDL-C, and high-density lipoprotein (HDL-C), were determined from blood samples. The Laboratory Center of Zhongda Hospital implements internal and external quality control procedures as directed by the Chinese Laboratory Quality Control.

2.4. Neuropsychological Tests. Neuropsychological tests, including the Montreal Cognitive Assessment (MoCA), Digit Span Test (DST), Verbal Fluency Test (VFT), Clock Drawing Test(CDT), Trail Making Test-A (TMTA), Trail Making Test-B (TMTB), Auditory Verbal Learning test-immediate recall (AVLT-IR), Auditory Verbal Learning test-delayed recall (AVLT-DR), and logical memory test (LMT) were administered to evaluate patients' cognitive functions, such as semantic memory, episodic memory, attention, executive function, and visuospatial skills. MoCA was used to assess all cognitive ability, and these patients with T2DM were divided into MCI $(<26)$ and non-MCI groups $(\geq 26)$.

2.5. DNA Isolation. Genomic DNA was isolated from blood samples (EDTA treated) according to the protocol of a DNA purification kit (Puregene, Gentra System, Minneapolis, MN) and similar with our previous studies [22]. It can be described briefly as follows: $1 \mathrm{ml}$ blood sample and $1 \mathrm{ml}$ cell lysate solution (CL) were mixed sufficiently. After thorough mixing, the mixture is centrifuged for 2 minutes at $3600 \mathrm{rpm}$. Then, the supernatant was removed before $1.5 \mathrm{ml}$ CL was added in the precipitate. The mixture was mixed and centrifuged for 2 minutes at $3600 \mathrm{rpm}$. Before $0.5 \mathrm{ml}$ mixture (proteinase $\mathrm{K}$ : buffer $\mathrm{PG}=1: 120$ ) was added in the precipitate, the supernatant was removed. The above mixture was vortex mixed immediately until the solution is free of lumps. And then, the mixture was incubated at $65^{\circ} \mathrm{C}$ for 30 minutes. After the mixture become green from red, $1 \mathrm{ml}$ isopropanol was added in the mixture and mixed sufficiently until enough filamentous DNA appeared in the centrifuge tubes. Tubes with DNA were centrifuged for 10 minutes at $3600 \mathrm{rpm}$ and then put them upside-down on clean absorbent paper for several minutes. After $1 \mathrm{ml} 75 \%$ alcohol was added, the tubes were vortex mixed for 5 seconds and centrifuged for 3 minutes at $3600 \mathrm{rpm}$ (this step was repeated 2 times). The tubes were put upside-down on a clean absorbent paper to dry for at least 5 minutes. One milliliter buffer TB was added to dissolve the DNA for $1 \mathrm{~h}$ at $65^{\circ} \mathrm{C}$ in the water bath. This dissolved DNA was used for further study.

2.6. INSIG-2 rs7566605 SNP Measurements. The following forward primer sequence and reverse primer sequence were used. Forward primer sequence: $5^{\prime}$-ACGTTGGATGTCAT TGCAATAGCCACTGCC- $3^{\prime}$, reverse primer sequence: $5^{\prime}$ -ACGTTGGATGAAAACCACCCTGGTACAGAC-3'. The DNA sequences of INSIG-2 were amplificated in a reaction system (containing $172.25 \mu \mathrm{l}$ of $25 \mathrm{mM} \mathrm{MgCl} 2,53 \mu \mathrm{l}$ of $25 \mathrm{mM}$ DNT Mix, $106 \mu \mathrm{l}$ of $5 \mathrm{U} / \mu \mathrm{l}$ HotStar Taq, $331.25 \mu \mathrm{l}$ of $\times 10$ PCR buffer, $10 \mathrm{ng} / \mu \mathrm{l}$ of 1 well genomic DNA, $530 \mu \mathrm{l}$ of $0.5 \mu \mathrm{mol} / 1$ primer, and $927.5 \mu \mathrm{l} \mathrm{HPLC}$ grade water) and initiated at $94^{\circ} \mathrm{C}$ for 2 minutes, followed by 45 cycles of 
TABLE 1: Demographic, clinical, and cognitive characteristics of T2DM patients with or without MCI.

\begin{tabular}{|c|c|c|c|}
\hline & MCI (87) & Non-MCI (146) & $P$ \\
\hline Age (year) & $60(55-68)$ & $59(53-65)$ & $0.046^{\ddagger *}$ \\
\hline Female (\%) & $44(50.6)$ & $47(32.2)$ & $0.005^{\S *}$ \\
\hline Education (year) & $9(9-12)$ & $11(9-12)$ & $0.004^{\ddagger *}$ \\
\hline BMI $\left(\mathrm{kg} / \mathrm{m}^{2}\right)$ & $24.486(22.758-27.041)$ & $24.671(22.740-26.298)$ & $0.730^{\ddagger}$ \\
\hline DM duration (year) & $12(8-16)$ & $10(6-15)$ & $0.025^{\ddagger *}$ \\
\hline HBP duration (year) & $8(0-17)$ & $1(0-10)$ & $0.005^{\ddagger *}$ \\
\hline Smoking (\%) & $26(29.9)$ & $56(38.4)$ & $0.211^{\S}$ \\
\hline Insulin usage (\%) & $50(57.5)$ & $86(58.9)$ & $0.783^{\S}$ \\
\hline Metformin usage (\%) & $51(58.6)$ & $100(68.5)$ & $0.127^{\S}$ \\
\hline HbAlc (\%) & $9.2(8.0-10.8)$ & $8.4(7.4-10.0)$ & $0.011^{\ddagger *}$ \\
\hline $\mathrm{FPG}(\mathrm{mmol} / \mathrm{l})$ & $7.80(6.41-10.20)$ & $7.60(6.59-9.72)$ & $0.382^{\ddagger}$ \\
\hline $\mathrm{TG}(\mathrm{mmol} / \mathrm{l})$ & $1.85(0.94-2.59)$ & $1.45(1.00-2.14)$ & $0.100^{\ddagger}$ \\
\hline $\mathrm{TC}(\mathrm{mmol} / \mathrm{l})$ & $4.72 \pm 1.08$ & $4.49 \pm 1.02$ & $0.149^{\dagger}$ \\
\hline $\operatorname{HDL}(\mathrm{mmol} / \mathrm{l})$ & $1.09(0.95-1.27)$ & $1.10(0.93-1.30)$ & $0.686^{\ddagger}$ \\
\hline $\mathrm{LDL}(\mathrm{mmol} / \mathrm{l})$ & $2.82(2.34-3.68)$ & $2.71(2.15-3.40)$ & $0.163^{\ddagger}$ \\
\hline ApoA1 & $1.13 \pm 0.29$ & $1.11 \pm 0.29$ & $0.480^{\dagger}$ \\
\hline ApoB & $0.80(0.66-0.90)$ & $0.80(0.64-0.96)$ & $0.518^{\ddagger}$ \\
\hline $\mathrm{LPa}$ & $156(83-315)$ & $126(70-270)$ & $0.246^{\ddagger}$ \\
\hline MoCA & $23(20-24)$ & $27(26-28)$ & $<0.001^{* *}$ \\
\hline DST & $11(9-12)$ & $12(11-14)$ & $<0.001^{\ddagger *}$ \\
\hline VFT & $14(13-17)$ & $17(14-21)$ & $<0.001^{\ddagger *}$ \\
\hline CDT & $3(2-4)$ & $4(3-4)$ & $<0.001^{\ddagger *}$ \\
\hline TMTA & $70(55-89)$ & $55(46-70)$ & $<0.001^{\ddagger *}$ \\
\hline TMTB & $187(142-255)$ & $131(100-173)$ & $<0.001^{\ddagger *}$ \\
\hline AVLT-IR & $16(13-18)$ & $19(16-23)$ & $<0.001^{\ddagger *}$ \\
\hline AVLT-DR & $5(3-6)$ & $6(5-8)$ & $<0.001^{\ddagger *}$ \\
\hline LMT & $8(4-10)$ & $11(8-14)$ & $<0.001^{\ddagger *}$ \\
\hline
\end{tabular}

BMI: body mass index; DM: diabetes mellitus; HBP: high blood pressure; FPG: fasting plasma glucose; TG: triglycerides; TC: total cholesterol; LDL: low-density lipoprotein cholesterol; HDL: high-density lipoprotein cholesterol; ApoA1: apolipoprotein A1; ApoB: apolipoprotein B; LPa: lipoprotein A; MoCA: Montreal Cognitive Assessment; DST: Digit Span Test; VFT: Verbal Fluency Test; CDT: Clock Drawing Test; TMTA: Trail Making Test-A; TMTB: Trail Making Test-B; AVLT-IR: Auditory Verbal Learning test-immediate recall; AVLT-DR: Auditory Verbal Learning test-delayed recall; LMT: logical memory test; MCI: individuals with mild cognitive impairment; Non-MCI: individuals without mild cognitive impairment. The data are presented as $n$ (\%), the mean \pm SD, or the median (inter-quartile range) unless otherwise specified. ${ }^{\dagger}$ Student's $t$ test was employed for normally distributed variables. ${ }^{*} T h e$ Mann-Whitney $U$ test

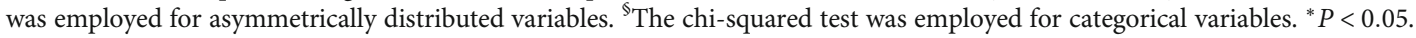

denaturation at $94^{\circ} \mathrm{C}$ for 20 seconds, $56^{\circ} \mathrm{C}$ for 30 seconds, and $72^{\circ} \mathrm{C}$ for 60 seconds and a final extension step at $72^{\circ} \mathrm{C}$ for 3 minutes. The above reaction products $(9 \mu \mathrm{l}$ in total) were diluted 3 times and desalinated with resin. Desalted samples were placed on the sample target to crystallize naturally. Finally, mass spectrometry detection was performed.

2.7. Statistical Analysis. Statistical analyses were conducted with SPSS 20.0 (SPSS Inc., Chicago, IL, USA). Student's $t$ test and one-way ANOVA were employed for normally distributed variables. The nonparametric Mann-Whitney $U$ and Kruskal-Wallis tests were used for asymmetrically distributed variables. The chi-squared test was utilized to test for binary variables. Partial correlation analysis and multiple linear regression analysis were carried out to explore the relationships between the cognitive measures and demographic characteristics. All statistical significance was defined as $P<0.05$.

\section{Results}

3.1. Demographic, Clinical, and Neuropsychological Data of T2DM Patients with or without MCI. In this cross-section study, demographic, clinical, and neuropsychological data were collected and are described in Table 1 . There are significant differences in age, gender, education level, DM duration, HBP duration, and HbA1c levels in T2DM with or without MCI (all $P<0.05$ ). However, we did not find significance between in BMI, smoking, insulin usage, metformin usage, FPG, TG, TC, HDL, LDL, ApoA1, ApoB, and LPa 
TABLE 2: Distributions of the INSIG-2 rs7566605 genotypes in T2DM patients with MCI or normal cognition.

\begin{tabular}{lcccc}
\hline Genotypes & $\begin{array}{c}\text { MCI } \\
(n, \%)\end{array}$ & $\begin{array}{c}\text { Non-MCI } \\
(n, \%)\end{array}$ & OR (95\% CL) & $P$ \\
\hline All & 87 & 146 & & \\
G & $114(65.5)$ & $165(56.5)$ & 1.000 & \\
C & $60(34.5)$ & $127(43.5)$ & $1.462(0.991-2.158)$ & 0.055 \\
GG & $38(43.7)$ & $51(34.9)$ & 1.000 & \\
GC & $38(43.7)$ & $63(43.2)$ & $1.235(0.690-2.210)$ & 0.476 \\
CC & $11(12.6)$ & $32(21.9)$ & $2.168(0.971-4.841)$ & 0.056 \\
GC+CC & $49(56.3)$ & $95(65.1)$ & $1.445(0.839-2.487)$ & 0.184 \\
\hline
\end{tabular}

MCI: individuals with mild cognitive impairment; Non-MCI: individuals without mild cognitive impairment. The genotypes and allele frequencies were compared between the groups using Pearson's $\chi^{2}$ tests.

levels (all $P>0.05$ ). As the important elements of cognition decline, MoCA, DST, VFT, CDT, AVLT-IR, AVLT-DR, and LMT scores decreased, while TMTA and TMTB scores increased in the MCI group, compared with the non-MCI group (all $P<0.05)$.

3.2. INSIG-2 rs7566605 Genotype Frequencies between the MCI and Non-MCI Groups. No significant differences in INSIG-2 rs7566605 genotype distributions were identified between the MCI and non-MCI groups (all $P>0.05$, Table 2).

3.3. Demographic, Clinical, and Neuropsychological Data of T2DM Patients with Different Genotypes. Although there was no significant difference of genotype distribution in T2DM patients with or without MCI, we found difference of metformin use frequencies, TC, LDL, and ApoB levels, as well as MoCA score and TMTB score (Table 3, all $P<0.05$ ), indicating the executive function. To further explore the role of INSIG-2 genotypes in MCI, the values above were compared between each two groups with different genotypes. Interestingly, compared to the GG group, there are decreased TC and LDL levels in the GC group; however, we also detected lower TC, LDL, and ApoB levels in the CC group than that in the GG and GC groups (all $P<0.05$ ). In addition, we measured a higher MoCA score in the $\mathrm{CC}$ group than the GC and GG groups (all $P<0.05$ ). Furthermore, there is a decreased TMTB score in the CC group, compared to the GG group $(P<0.05)$ (Table 4$)$.

3.4. Associations between TC, LDL and ApoB and Neuropsychological Test Scores. INSIG-2 is an important gene associated with cholesterol metabolism. Improved cognition was found in CC genotype patients, compared with patients with GG and GC genotypes. To further investigate the potential mechanism between INSIG-2 SNP and cognition decline, a partial correlation was administrated. TC and LDL are associated with TMTB score adjusted by age, education level, gender, DM duration, HBP duration, smoking, insulin usage, and metformin usage $(R=0.145, P=0.030$, and $R=0.193$, $P=0.004$, respectively) (Table 5).
3.5. Multiple Linear Regression Analysis with TMTB as the Dependent Variable. To further explore the potential role of TC and LDL in the cognition of T2DM patients, multiple linear regression analysis was performed. Corrected with age, education level, gender, DM duration, HBP duration, smoking, insulin usage, and metformin usage, TC and LDL are associated with TMTB, which is an index associated with executive function $(\beta=8.146, P=0.030$, and $\beta=13.704$, $P=0.005$, respectively) (Table 6 ).

\section{Discussion}

For the increased T2DM prevalence [1, 23], uncontrolled plasma glucose is a risk factor of $\mathrm{AD}$ and MCI in our previous studies [24-26] and others [27]. In the present study, our results supported these conclusions by discovering the increased HbA1c level of T2DM patients with MCI, compared to those without MCI. Although we found cognition decline in T2DM patients with hypercholesteremia in our previous study [8], and higher cholesterol levels in T2DM patients with MCI than those without MCI in the present research, the difference between levels of TC $(4.72 \pm 1.08$ vs. $4.49 \pm 1.02)$ or $\operatorname{LDL}(2.82,2.34-3.68$, vs. $2.71,2.15-3.40)$ is not statistically significant. This may account for the limited participations. In addition, more and more research suggested that hypercholesteremia is a risk factor of cognitive impairment in several high-fat diet-induced or genetic hypercholesteremia animal studies [28-30] and in clinical trials $[31,32]$.

While the important role of cholesterol in the process of cognition decline is well recognized $[33,34]$, the role of cholesterol and the genetic factors regulating the metabolism of cholesterol in cognitive impairment with T2DM remains undiscovered. INSIG-2 is a sensor and mediator that regulates the transcription and translation of HMG-CoA reductase and LDL receptor to keep cholesterol, especially LDL homeostasis [11]. The potential role of INSIG-2 gene involved in T2DM with MCI is still unclear. Moreover, the promoter of INSIG-2 rs7566605 SNP is associated with the prevalence of hypercholesterolemia [16]. Additionally, the CC genotype is an independent protective genetic factor for the progressing hypercholesterolemia induced by high-fat diet, especially in female subjects [17]. So, in this work, these genotypes of the participants were measured. We found more $\mathrm{CC}$ and less GG genotypes in T2DM patients without MCI than those with MCI, but there is no statistical significance $(P=0.056)$.

To further explore the relationship among INSIG-2 rs7566605 SNP, hypercholesterolemia, and cognitive impairment, the level of demographic and clinical data and neuropsychological tests results were compared. Interestingly, we detected significant differences in TC, LDL, and ApoB levels as well as MoCA and TMTB scores among the three genotypes. In addition, decreased TC, LDL, and ApoB levels were observed in patients with $\mathrm{CC}$ genotype than these with GC or GG genotype. Moreover, increased MoCA score and decreased TMTB score were tested in patients with CC genotype, compared with those with GC or GG genotype. The above conclusions suggested that unbalanced TC and 
TABle 3: Demographic, clinical, and cognitive characteristics of T2DM patients with different genotypes.

\begin{tabular}{|c|c|c|c|c|}
\hline & GG (101) & GC (89) & CC (43) & $P$ \\
\hline Age (year) & $60(55-67)$ & $60(53-67)$ & $56(52-64)$ & $0.210^{\ddagger}$ \\
\hline Female (\%) & $36(40.4)$ & $35(34.7)$ & $20(46.5)$ & $0.442^{\S}$ \\
\hline Education (year) & $10(9-12)$ & $11(9-12)$ & $10(9-12)$ & $0.914^{\ddagger}$ \\
\hline $\operatorname{BMI}\left(\mathrm{kg} / \mathrm{m}^{2}\right)$ & $24.93(22.87-27.09)$ & $14.09(22.08-26.21)$ & $24.62(23.60-25.88)$ & $0.318^{\ddagger}$ \\
\hline DM duration (year) & $10(6-15)$ & $10(8-16)$ & $10(6-15)$ & $0.768^{\ddagger}$ \\
\hline HBP duration (year) & $6(0-16)$ & $3(0-10)$ & $1(0-11)$ & $0.230^{\ddagger}$ \\
\hline Smoking (\%) & $33(37.1)$ & $36(35.6)$ & $13(30.2)$ & $0.737^{\S}$ \\
\hline Insulin usage (\%) & $48(53.9)$ & $60(59.4)$ & $28(65.1)$ & $0.442^{\S}$ \\
\hline Metformin usage (\%) & $56(62.9)$ & $59(58.4)$ & $36(83.7)$ & $0.013^{\S *}$ \\
\hline HbAlc (\%) & $8.6(7.6-10.6)$ & $8.7(7.8-10.5)$ & $8.6(7.7-10.2)$ & $0.797^{\ddagger}$ \\
\hline FPG (mmol/l) & $7.40(6.35-10.00)$ & $7.70(6.61-9.47)$ & $8.00(6.67-10.20)$ & $0.257^{\ddagger}$ \\
\hline $\mathrm{TG}(\mathrm{mmol} / \mathrm{l})$ & $1.48(1.03-2.41)$ & $1.52(0.90-2.67)$ & $1.67(1.08-2.25)$ & $0.734^{\ddagger}$ \\
\hline $\mathrm{TC}(\mathrm{mmol} / \mathrm{l})$ & $4.93 \pm 1.06$ & $4.53 \pm 1.20$ & $3.94 \pm 1.02$ & $<0.001^{\dagger *}$ \\
\hline $\mathrm{HDL}(\mathrm{mmol} / \mathrm{l})$ & $0.93(1.09-1.31)$ & $1.10(0.99-1.28)$ & $1.03(0.87-1.27)$ & $0.341^{\ddagger}$ \\
\hline LDL (mmol/l) & $2.96(2.50-3.70)$ & $2.77(2.22-3.53)$ & $2.14(1.76-2.71)$ & $<0.001^{\text {f* }}$ \\
\hline ApoA1 & $1.12 \pm 0.28$ & $1.11 \pm 0.28$ & $1.13 \pm 0.33$ & $0.869^{\dagger}$ \\
\hline ApoB & $0.82(0.71-0.97)$ & $0.81(0.64-1.01)$ & $0.72(0.59-0.85)$ & $0.005^{\ddagger *}$ \\
\hline $\mathrm{LPa}$ & $143(69-285)$ & $149(79-318)$ & $111(70-227)$ & $0.339^{\ddagger}$ \\
\hline MoCA & $26(22-27)$ & $26(24-27)$ & $27(25-28)$ & $0.027^{\ddagger *}$ \\
\hline DST & $12(11-13)$ & $11(10-13)$ & $12(11-13)$ & $0.158^{*}$ \\
\hline VFT & $15(13-18)$ & $16(14-19)$ & $16(14-20)$ & $0.123^{\ddagger}$ \\
\hline $\mathrm{CDT}$ & $4(3-4)$ & $4(3-4)$ & $4(3-4)$ & $0.082^{\ddagger}$ \\
\hline TMTA & $61(50-101)$ & $58(48-71)$ & 57 (047-79) & $0.109^{\ddagger}$ \\
\hline TMTB & $160(119-252)$ & $148(115-189)$ & $128(80-195)$ & $0.016^{\ddagger *}$ \\
\hline AVLT-IR & $17(14-21)$ & $18(15-21)$ & $18(15-23)$ & $0.391^{\text {* }}$ \\
\hline AVLT-DR & $5(4-7)$ & $6(5-7)$ & $6(05-8)$ & $0.144^{\ddagger}$ \\
\hline LMT & $9.58 \pm 4.76$ & $9.24 \pm 4.10$ & $10.27 \pm 4.68$ & $0.461^{\dagger}$ \\
\hline
\end{tabular}

BMI: body mass index; DM: diabetes mellitus; HBP: high blood pressure; FPG: fasting plasma glucose; TG: triglycerides; TC: total cholesterol; LDL: low-density lipoprotein cholesterol; HDL: high-density lipoprotein cholesterol; ApoA1: apolipoprotein A1; ApoB: apolipoprotein B; LPa: lipoprotein A; MoCA: Montreal Cognitive Assessment; DST: Digit Span Test; VFT: Verbal Fluency Test; CDT: Clock Drawing Test; TMTA: Trail Making Test-A; TMTB: Trail Making Test-B; AVLT-IR: Auditory Verbal Learning test-immediate recall; AVLT-DR: Auditory Verbal Learning test-delayed recall; LMT: logical memory test; MCI: individuals with mild cognitive impairment; Non-MCI: individuals without mild cognitive impairment. The data are presented as $n$ (\%), the mean \pm SD, or the median (interquartile range) unless otherwise specified. ${ }^{\dagger}$ One-way ANOVA was employed for normally distributed variables. ${ }^{\ddagger}$ The Kruskal-Wallis test was employed for asymmetrically distributed variables. ${ }^{5} \mathrm{The}$ chi-squared test was employed for categorical variables. ${ }^{*} P<0.05$.

TABle 4: TC, LDL, ApoB, MoCA, adjusted MoCA, and TMTB in two different genotypes.

\begin{tabular}{lccc}
\hline & GC vs. GG & CC vs. GC & CC vs. GG \\
& $P$ & $P$ & $P$ \\
\hline TC & $0.013^{\dagger *}$ & $0.004^{\dagger *}$ & $<0.001^{\dagger *}$ \\
LDL & $0.025^{\ddagger *}$ & $0.002^{\ddagger *}$ & $<0.001^{\ddagger *}$ \\
ApoB & $0.340^{\ddagger}$ & $0.010^{\ddagger *}$ & $0.001^{\ddagger *}$ \\
MoCA & $0.613^{\ddagger}$ & $0.024^{\ddagger *}$ & $0.009^{\ddagger *}$ \\
TMTB & $0.108^{\ddagger}$ & $0.109^{\ddagger}$ & $0.005^{\ddagger *}$ \\
\hline
\end{tabular}

TC: total cholesterol; LDL: low-density lipoprotein cholesterol; ApoB: apolipoprotein B; MoCA: Montreal Cognitive Assessment; TMTB: Trail Making Test-B. ${ }^{\dagger}$ One-way ANOVA was employed for normally distributed variables. ${ }^{*}$ The Kruskal-Wallis test was employed for asymmetrically distributed variables. ${ }^{*} P<0.05$.
TABLE 5: Relationships between TC, LDL, and ApoB levels and neuropsychological test scores.

\begin{tabular}{ccccccc}
\hline & \multicolumn{2}{c}{ Model $^{1}$} & \multicolumn{2}{c}{ Model $^{2}$} & \multicolumn{2}{c}{ Model $^{3}$} \\
& $R$ & $P$ & $R$ & $P$ & $R$ & $P$ \\
\hline MoCA & -0.069 & 0.350 & -0.072 & 0.286 & -0.052 & 0.437 \\
TMTB & 0.145 & $0.030^{*}$ & 0.193 & $0.004^{*}$ & 0.021 & 0.750 \\
\hline
\end{tabular}

${ }^{1}$ Correlation between TC and MoCA (or TMTB) adjustment by age, education level, gender, DM duration, HBP duration, smoking, insulin usage, and metformin usage. ${ }^{2}$ Correlation between LDL and MoCA (or TMTB) adjustment by age, education level, gender, DM duration, HBP duration, smoking, insulin usage, and metformin usage. ${ }^{3}$ Correlation between ApoB and MoCA (or TMTB) adjustment by age, education level, gender, DM duration, HBP duration, smoking, insulin usage, and metformin usage. TC: total cholesterol; LDL: low-density lipoprotein cholesterol; ApoB: apolipoprotein B; MoCA: Montreal Cognitive Assessment; TMTB: Trail Making Test-B. ${ }^{*} P<0.05$. 
TABLE 6: Multiple linear regression analysis of the factors influencing TMTB.

\begin{tabular}{lcccc}
\hline & \multicolumn{2}{c}{ Model $^{1}$} & \multicolumn{2}{c}{ Model $^{2}$} \\
& $\beta$ & $P$ & $\beta$ & $P$ \\
\hline Age & 1.670 & $0.006^{*}$ & 1.693 & $0.005^{*}$ \\
Gender & 23.573 & $0.031^{*}$ & 23.756 & $0.027^{*}$ \\
Education & -4.996 & $0.001^{*}$ & -5.097 & $<0.001^{*}$ \\
BMI & -1.730 & 0.215 & -1.777 & 0.198 \\
DM duration & 0.454 & 0.557 & 0.388 & 0.630 \\
HBP duration & 1.221 & 0.009 & 1.263 & 0.009 \\
Smoking & -0.592 & 0.957 & -0.252 & 0.981 \\
Insulin usage & -9.854 & 0.270 & -9.314 & 0.293 \\
Metformin usage & -8.651 & 0.362 & -9.832 & 0.295 \\
TC or LDL & 8.416 & $0.030^{*}$ & 13.704 & $0.005^{*}$ \\
\hline
\end{tabular}

${ }^{1}$ Independent variables entered included age, education level, gender, DM duration, HBP duration, smoking, insulin usage, metformin usage, and TC. ${ }^{2}$ Independent variables entered included age, education level, gender, DM duration, HBP duration, smoking, insulin usage, metformin usage, and LDL. TC: total cholesterol; LDL: low-density lipoprotein cholesterol; BMI: body mass index; DM: diabetes mellitus; HBP: high blood pressure. ${ }^{*} P<0.05$.

LDL result from ApoB and INSIG-2 rs7566605 SNP may be associated with cognitive dysfunction of T2DM.

To investigate the association among TC, LDL, ApoB, and cognitive dysfunction, correlation analysis between these above cholesterol metabolic indexes and MoCA (or TMTB) scores was performed. Although we did not find a correlation between MoCA (or TMTB) and ApoB, significant correlations were found between TC or LDL and TMTB scores ( $R=0.145, P=0.030$, or $R=0.193, P=0.004$, respectively), indicating the executive function [35]. Moreover, multiple linear regression with TMTB score insisted that TC and LDL are associated with the TMTB score independent from age, gender, education, BMI, DM duration, HBP duration, smoking, insulin, and metformin usage.

Overall, the INSIG-2 rs7566605 SNP may be associated with cognitive decline. In other words, CC genotype has a neuroprotective effect on T2DM patients, especially for executive function. In addition, cholesterol homeostasis, especially for LDL, may be involved in these mechanisms.

In this study, we demonstrated lower TC and LDL levels and TMTB score in patients with CC genotype than those with GC or GG genotype. However, there is no significant difference of TMTB scores between patients with CC genotype and GC genotype $(P=0.109)$, due to the limited number of participants listed in this study. The present research showed decreased TC, LDL levels, and TMTB in the CC genotype, compared to those with other genotypes. Although previous studies suggested that INSIG-2 rs7566605 SNP is associated with hypercholesteremia, we did not get the direct association between INSIG-2 rs7566605 SNP and MCI. So, we just guess that CC genotypes may have the neuroprotective effects on T2DM patients. Furthermore, we only observed genotypes in this research; the expression level of INSIG-2 was not detected. In our following study, we would like to measure the global expression level of INSIG-2 in human and local INSIG-2 in animal models. Last, our study is a cross-sectional study, which can only explain the association between INSIG-2 genotype and mild cognitive impairment but fails to explain the causal relationship between them. The causal relationship between them needs to be further validated by a cohort study.

\section{Data Availability}

All data used to support the findings of this study are available from the corresponding author upon request.

\section{Ethical Approval}

Trial registration number: ChiCTROCC15006060.

\section{Conflicts of Interest}

The authors declare that they have no competing interests.

\section{Authors' Contributions}

SW and HZ designed the study. HZ, RH, ST, KA, WZ, JS, and WC collected the data, extracted the DNA from the samples, and performed statistical analysis. $\mathrm{HZ}$ wrote the manuscript draft. SW helped to revise the final version of paper. All authors have read and approved the final manuscript.

\section{Acknowledgments}

This work was partially supported by the National Natural Science Foundation of China (No. 81570732, Shaohua Wang, and No. 81870568, Shaohua Wang). INSIG-2 rs7566605 SNP was measured by the Beijing Cnkingbio Biotechnology Corporation (http://www.cnkingbio.com/).

\section{References}

[1] L. Wang, P. Gao, M. Zhang et al., "Prevalence and ethnic pattern of diabetes and prediabetes in China in 2013," JAMA, vol. 317, no. 24, pp. 2515-2523, 2017.

[2] G. J. Biessels, S. Staekenborg, E. Brunner, C. Brayne, and P. Scheltens, "Risk of dementia in diabetes mellitus: a systematic review," The Lancet Neurology, vol. 5, no. 1, pp. 64-74, 2006.

[3] E. Mariani, R. Monastero, and P. Mecocci, "Mild cognitive impairment: a systematic review," Journal of Alzheimer's disease, vol. 12, no. 1, pp. 23-35, 2007.

[4] S. Tian, J. Han, R. Huang et al., "Increased plasma homocysteine level is associated with executive dysfunction in type 2 diabetic patients with mild cognitive impairment," Journal of Alzheimer's disease : JAD, vol. 58, no. 4, pp. 1163-1173, 2017.

[5] S. Tian, J. Han, R. Huang et al., "Association of increased serum ACE activity with logical memory ability in type 2 diabetic patients with mild cognitive impairment," Frontiers in Behavioral Neuroscience, vol. 10, p. 239, 2016.

[6] M. Kivipelto and A. Solomon, "Cholesterol as a risk factor for Alzheimer's disease - epidemiological evidence," Acta Neurologica Scandinavica, vol. 114, no. s185, pp. 50-57, 2006.

[7] A. R. Koudinov and N. V. Koudinova, "Cholesterol homeostasis failure as a unifying cause of synaptic degeneration," 
Journal of the Neurological Sciences, vol. 229-230, pp. 233-240, 2005.

[8] W. Xia, B. Zhang, Y. Yang, P. Wang, Y. Yang, and S. Wang, "Poorly controlled cholesterol is associated with cognitive impairment in T2DM: a resting-state fMRI study," Lipids in Health and Disease, vol. 14, no. 1, p. 47, 2015.

[9] S.-h. Wang, Y. Huang, Y. Yuan, W.-q. Xia, P. Wang, and R. Huang, "LDL receptor knock-out mice show impaired spatial cognition with hippocampal vulnerability to apoptosis and deficits in synapses," Lipids in Health and Disease, vol. 13, no. 1, p. 175, 2014.

[10] Y. Peng, E. J. Schwarz, M. A. Lazar, A. Genin, N. B. Spinner, and R. Taub, "Cloning, human chromosomal assignment, and adipose and hepatic expression of the CL-6/INSIG1 gene," Genomics, vol. 43, no. 3, pp. 278-284, 1997.

[11] D. Yabe, M. S. Brown, and J. L. Goldstein, "Insig-2, a second endoplasmic reticulum protein that binds SCAP and blocks export of sterol regulatory element-binding proteins," Proceedings of the National Academy of Sciences of the United States of America, vol. 99, no. 20, pp. 12753-12758, 2002.

[12] R. Y. Hampton, "Proteolysis and sterol regulation," Annual Review of Cell and Developmental Biology, vol. 18, no. 1, pp. 345-378, 2002.

[13] A. Herbert, N. P. Gerry, M. B. McQueen et al., "A common genetic variant is associated with adult and childhood obesity," Science, vol. 312, no. 5771, pp. 279-283, 2006.

[14] H. J. Wang, H. Zhang, S. W. Zhang, Y. P. Pan, and J. Ma, "Association of the common genetic variant upstream of INSIG2 gene with obesity related phenotypes in Chinese children and adolescents," Biomedical and environmental sciences : BES, vol. 21, no. 6, pp. 528-536, 2008.

[15] D. H. Hall, T. Rahman, P. J. Avery, and B. Keavney, "INSIG-2 promoter polymorphism and obesity related phenotypes: association study in 1428 members of 248 families," BMC Medical Genetics, vol. 7, no. 1, p. 83, 2006.

[16] K. Oki, K. Yamane, N. Kamei, T. Asao, T. Awaya, and N. Kohno, "The single nucleotide polymorphism upstream of insulin-induced gene 2 (INSIG2) is associated with the prevalence of hypercholesterolaemia, but not with obesity, in Japanese American women," The British Journal of Nutrition, vol. 101, no. 3, pp. 322-327, 2009.

[17] P. Zavattari, A. Loche, P. Civolani et al., "An INSIG2 polymorphism affects glucose homeostasis in Sardinian obese children and adolescents," Annals of Human Genetics, vol. 74, no. 5, pp. 381-386, 2010.

[18] M. A. Pappolla, T. K. Bryant-Thomas, D. Herbert et al., "Mild hypercholesterolemia is an early risk factor for the development of Alzheimer amyloid pathology," Neurology, vol. 61, no. 2, pp. 199-205, 2003.

[19] K. G. M. M. Alberti, P. Z. Zimmet, and W. H. O. Consultation, "Definition, diagnosis and classification of diabetes mellitus and its complications. Part 1: diagnosis and classification of diabetes mellitus provisional report of a WHO consultation," Diabetic Medicine, vol. 15, no. 7, pp. 539-553, 1998.

[20] F. Portet, P. J. Ousset, P. J. Visser et al., "Mild cognitive impairment (MCI) in medical practice: a critical review of the concept and new diagnostic procedure. Report of the MCI Working Group of the European Consortium on Alzheimer's Disease," Journal of Neurology, Neurosurgery, and Psychiatry, vol. 77, no. 6, pp. 714-718, 2006.
[21] P. Wang, R. Huang, S. Lu et al., "RAGE and AGEs in mild cognitive impairment of diabetic patients: a cross-sectional study," PLoS One, vol. 11, no. 1, article e0145521, 2016.

[22] R. Cai, J. Han, J. Sun et al., "Effects of ABCA1 R219K polymorphism and serum lipid profiles on mild cognitive impairment in type 2 diabetes mellitus," Frontiers in Aging Neuroscience, vol. 9, p. 257, 2017.

[23] Y. Xu, L. Wang, J. He et al., "Prevalence and control of diabetes in Chinese adults," JAMA, vol. 310, no. 9, pp. 948-959, 2013.

[24] R. J. McCrimmon, C. M. Ryan, and B. M. Frier, "Diabetes and cognitive dysfunction," The Lancet, vol. 379, no. 9833, pp. 2291-2299, 2012.

[25] S. L. C. Geijselaers, S. J. S. Sep, C. D. A. Stehouwer, and G. J. Biessels, "Glucose regulation, cognition, and brain MRI in type 2 diabetes: a systematic review," The Lancet Diabetes of endocrinology, vol. 3, no. 1, pp. 75-89, 2015.

[26] G. J. Biessels, I. J. Deary, and C. M. Ryan, "Cognition and diabetes: a lifespan perspective," The Lancet Neurology, vol. 7, no. 2, pp. 184-190, 2008.

[27] S. Tian, R. Huang, J. Han et al., "Increased plasma interleukin1 beta level is associated with memory deficits in type 2 diabetic patients with mild cognitive impairment," Psychoneuroendocrinology, vol. 96, pp. 148-154, 2018.

[28] R. Mastrocola, M. Guglielmotto, C. Medana et al., "Dysregulation of SREBP2 induces BACE1 expression," Neurobiology of Disease, vol. 44, no. 1, pp. 116-124, 2011.

[29] E. C. Cope, E. A. LaMarca, P. K. Monari et al., "Microglia play an active role in obesity-associated cognitive decline," The Journal of neuroscience, vol. 38, no. 41, pp. 8889-8904, 2018.

[30] W. D. Porter, P. R. Flatt, C. Holscher, and V. A. Gault, "Liraglutide improves hippocampal synaptic plasticity associated with increased expression of Mash1 in ob/ob mice," International Journal of Obesity, vol. 37, no. 5, pp. 678-684, 2013.

[31] H. Jick, G. L. Zornberg, S. S. Jick, S. Seshadri, and D. A. Drachman, "Statins and the risk of dementia," Lancet, vol. 356, no. 9242, pp. 1627-1631, 2000.

[32] C. S. Chu, P. T. Tseng, B. Stubbs et al., "Use of statins and the risk of dementia and mild cognitive impairment: a systematic review and meta-analysis," Scientific Reports, vol. 8, no. 1, p. $5804,2018$.

[33] F. Arenas, C. Garcia-Ruiz, and J. C. Fernandez-Checa, "Intracellular cholesterol trafficking and impact in neurodegeneration," Frontiers in Molecular Neuroscience, vol. 10, p. 382, 2017.

[34] K. J. Anstey, K. Ashby-Mitchell, and R. Peters, "Updating the evidence on the association between serum cholesterol and risk of late-life dementia: review and meta-analysis," Journal of Alzheimer's disease, vol. 56, no. 1, pp. 215-228, 2017.

[35] J. Wang, Y. Yuan, R. Cai et al., “Association between plasma levels of PAI-1, tPA/PAI-1 molar ratio, and mild cognitive impairment in Chinese patients with type 2 diabetes mellitus," Journal of Alzheimer's disease, vol. 63, no. 2, pp. 835-845, 2018. 